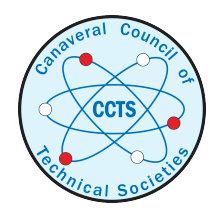

The Space Congress $®$ Proceedings

1970 (7th) Technology Today and Tomorrow

Apr 1st, 8:00 AM

\title{
Image Enhancement Techniques
}

Donald B. Gennery

Senior Systems Analyst, RCA MTP, Patrick AFB, Florida

Follow this and additional works at: https://commons.erau.edu/space-congress-proceedings

\section{Scholarly Commons Citation}

Gennery, Donald B., "Image Enhancement Techniques" (1970). The Space Congress ${ }^{\circledR}$ Proceedings. 4.

https://commons.erau.edu/space-congress-proceedings/proceedings-1970-7th/session-12/4

This Event is brought to you for free and open access by the Conferences at Scholarly Commons. It has been accepted for inclusion in The Space Congress ${ }^{\circledR}$

Proceedings by an authorized administrator of Scholarly Commons. For more information, please contact commons@erau.edu.

EMBRYRIDDLE Aeronautical University SCHOLARLY COMMONS 


\section{IMAGE ENHANCEMENT TECHNIQUES}

Donald B. Gennery

Senior Systems Analyst

RCA MTP

Patrick AFB, Florida

\section{ABSTRACT}

This paper discusses the enhancement of images to restore detail that has been obscured by blurring. An optical analog method and several digital methods of performing this processing are discussed. Sample results from one of the digital methods are shown.

\section{INTRODUCT ION}

In many fields of science and technology it often happens that a photograph has been obtained in such a manner that blurring has reduced the sharpness of the image. For example, in astronomical photographs obtained by earth-based telescopes, the turbulence of the atmosphere causes a degrading of the image. Similar effects occur in underwater photography. Also, in photographs obtained from space probes, improper focus or motion during the exposure may blur the image. In medical $X$-rays the resolution often is not as good as might be desired. Many other examples could be mentioned.

In cases such as the above, it sometimes is possible to process the image in such a way as to correct for the blurring process and thus to improve the resolution of the image. Other processing can also be performed so as to accentuate certain features of the image. As many others have pointed out $(1-6)$, these types of processing in many cases can be performed by twodimensional spatial filters. Both analog filters using optical techniques and digital filters using general-purpose digital computers will be discussed below.

\section{GENERAL PRINCIPLES}

If an image formed in noncoherent light is blurred in a constant way throughout the image plane, this smearing out process can be described by the convolution operation, as follows:

$$
z=g * \hat{z}
$$

where $\hat{z}$ is the ideal image intensity, $z$ is the actual image intensity, and $g$ is the point spread function of the blurring operation. (The quantities $z, \hat{z}$, and $g$ are al1 implicit functions of the two spatial coordinates, and the symbol * represents the two-dimensional convolution.)

Equation (1) can be transformed into the frequency domain, where the convolution becomes a multiplication by means of the convolution theorem, as follows:

$\mathrm{Z}=\mathrm{G} \hat{\mathrm{Z}}$

(A capital letter $\mathrm{Z}, \mathrm{G}, \mathrm{H}$, or $\mathrm{U}$ denotes the two-dimensional Fourier transform of the corresponding lower-case letter and represents a quantity that is a function of the two components of spatial frequency.) $G$ is called the optical transfer function or frequency response of the blurring operation. Equation (2) can be solved for $\mathrm{Z}$ as follows:

$\hat{Z}=\frac{Z}{G}$

Thus processing the blurred image with a filter whose transfer function is $1 / G$ would produce a restoration of the ideal image.

However, $G$ may be zero at certain frequencies. Furthermore, G may be so small at some frequencies that the image content at these frequencies has dropped below the noise level in the photographic emulsion and thus cannot be restored. Therefore, instead of equation (3) the following procedure is used.

The optimum filter for separating a message whose power spectrum is $\mathrm{P}_{\mathrm{m}}$ from noise whose power spectrum is $\mathrm{P}_{n}$ has the transfer function $\mathrm{P}_{m} /\left(\mathrm{P}_{m}+\mathrm{P}_{n}\right)$ if the message and noise are uncorrelated (7). The message in this case is the blurred image $z$, whose power spectrum is $P_{\hat{z}}|G|^{2}$, where $\mathrm{P}_{\hat{z}}$ is the power spectrum of the ideal image $\hat{z}$. Making this substitution and multiplying by the above function $1 / G$ for restoring the image in the absence of 
noise produces the following transfer function of the restoring filter including noise suppression:

$H=\frac{P_{\hat{z}}|G|^{2}}{P_{\hat{z}}|G|^{2}+P_{n}} \cdot \frac{1}{G}$

This reduces to

$H=\frac{\bar{G}}{|G|^{2}+B^{2}}$

where $\beta^{2}=P_{n} / P_{\hat{z}}$ and - denotes the complex conjugate. ( $\beta$ represents the ratio of noise amplitude to signal amplitude in the intensity data. $\beta$ in general is a function of the two components of spatial frequency, but it will be considered to be constant here for simplicity. Note that when $\beta=0, H=1 / G$.) Thus, instead of equation (3), we have

$\hat{\mathrm{Z}}=\mathrm{HZ}$

In the space domain, equation (6) becomes the following:

$\hat{\mathrm{z}}=\mathrm{h} * \mathrm{z}$

where $h$ is called the filter impulse response.

The filtering of an image to perform the above can be done by one of two basic methods. In the transform method, the input image $z$ is transformed to the frequency domain, multiplied by the filter transfer function $\mathrm{H}$ at each frequency according to equation $(6)$, and then transformed back to the space domain to produce the output image $z$. In the convolution method, the input image $z$ is convolved with the filter impulse response $h$ in the space domain according to equation (7) to produce the output image $\hat{z}$. (If either $h$ or $H$ is known, the other can be obtained by performing the appropriate two-dimensional Fourier transform.)

\section{DETERMINATION OF OPTICAL TRANSFER FUNCTION}

We must now discuss means of determining the optical transfer function $G$ of the blurring process so that it can be used in equation (4) to determine the restoring filter.

In some cases the optical transfer function can be computed from theoretical considerations. For example, if the blurring is caused by aberrations in lenses, the point spread function $\mathrm{g}$ can be computed by ray tracing, and the Fourier transform of this can be computed numerically to obtain the transfer function $G$. In many cases, however, the smearing is due to some phenomenon (such as unknown inhomogeneities in the medium) whose effects cannot be predicted and thus must be measured.
If a known object can be photographed along with the object under study, its blurred image can be used to determine the optical transfer function. If the ideal image of the known object is denoted by $\hat{u}$ and its blurred image by $u$, then

$\mathrm{u}=\mathrm{g} * \hat{\mathrm{u}}$

$\mathrm{U}=\mathrm{GU}$

Thus $G$ can be determined as follows, except where $\hat{\mathrm{U}}$ becomes so small as to be seriously contaminated by noise:

$G=\frac{U}{\hat{U}}$

A special case of equation (10) that is of some importance occurs when the known object is a point source. Then $\hat{u}$ is a delta function (unit impulse), and if we assume that it is at the origin, 0 is a cunstant. Furthermore, by normalizing U so that is is equal to unity at zero frequency, Û can be set equal to unity. Thus $\mathrm{G}=\mathrm{U}$ in this case. That is, the optical transfer function is equal to the twodimensional Fourier transform (normalized to be unity at the origin) of the blurred image of a point source. An example of using a point source as a standard can occur in astronomical photography, where a star can be considered to be a point source.

There are cases in which the optical transfer function can be partially computed from theoretical considerations, so that an analytical expression is obtained containing some unknown coefficients.

Such a case sometimes occurs with atmospheric turbulence, for example $e^{(5)}$. If a measurement of the transfer function is also obtained by using a known object as described above, the theoretical function can be fit to this measured data. That is, a least-squares (or other) solution for the unknown coefficients can be performed, and by using these values the theoretical and by using these values then be used. Using this adjusted theoretical function instead of the raw measured function reduces the effects of noise in the latter.

A fairly common simple case of the above use of a combination of theoretical know1. edge and measured data occurs when the entire shape of the optical transfer function is known theoretically and only the frequency scale factor is unknown. In this case just the scale factor needs to be determined in the adjustment. For example, in some cases it can be assumed with good accuracy that the optical trans fer function is a Gaussian function, and only its width needs to be determined. (Due to the Central Limit Theorem in statistics, many random processes tend to produce the Gaussian function.) 
If the shape of the optical transfer function is known and only its frequency scale factor needs to be determined, a trial and error procedure can often be used. This would be done by using the filter transfer function computed from equation (5) but adjusted for a series of different scale factors. When the blurred image is processed by each of these filters, it should be possible to determine the best one by visual inspection of the results to see which filtered image has been sharpened up the best. While this procedure may be time consuming, it requires no specific information about the content of the image, but is based only on general information about the presence of sharp detail or types of shapes in the ideal image.

\section{ANALOG TECHNIQUES}

Methods of performing the above processing by means of analog computations using coherent light wil1 now be discussed. The transform method of filtering an image will be used, as stated by equation (6).

The complex amplitude distribution in the back focal plane of an ideal lens is the Fourier transform of that in the front focal plane $(8)$. This fact is utilized to perform the two Fourier transforms needed in the transform method.

The basic configuration is shown in Figure 1. A positive transparency of the image to be filtered is inserted into the front focal plane of lens 1 of focal length $L$ and is illuminated with collimated coherent light. This transparency must have a gamma of 2 so that the amplitude of 1 ight passing through it is proportional to the intensity in the original image. The spatial frequencies of the original image are displayed in the back focal plane of the lens. Here a filter is positioned, whose amplitude transmittance at each position is proportional to the desired transfer function $\mathrm{H}$ at the corresponding frequency. Thus the multiplication called for by equation (6) is performed. The filter plane is also the front focal plane of a second lens of focal length $L$, which transforms the result back to the space domain. The resulting filtered image appears in the back focal plane of this lens. (The frequency scale factor in the back focal plane of lens 1 , that is, the ratio of spatial coordinate in the back focal plane to frequency component in the front focal plane, is $L \lambda$, where $\lambda$ is the wavelength.)

If the function $H$ is always positive real, the above method is satisfactory. However, if $\mathrm{H}$ contains imaginary parts, the filter must produce phase shift in addition to changing the amplitude. One way of doing this is to use a $\mathrm{film}$ of varying thickness. Such a method involves considerable practical difficulties, however, and a more suitable method of controliing the phase is to convert the phase information into amplitude information by modulating the desired transfer function $\mathrm{H}$ onto a carrier.

The carrier consists of a sinusoidal pattern of amplitude transmission bands proceeding in one direction across the filter. The amplitude of the carrier wave is proportional to the absolute value of $\mathrm{H}$, and the phase of the carrier is determined by the phase (argument) of $\mathrm{H}$. The filter transparency then is one type of hologram $(9)$. The carrier in the hologram acts iike a diffraction grating, and the first-order light diffracted in the proper direction to produce the correct polarity of phase shift produces the desired filtered image. The undiffracted 1 ight and the light diffracted in the other direction produce unwanted images that can be ignored if they are sufficiently separated. Higher-order diffracted images will not exist if the carrier wave is truly sinusoidal. This entire process is illustrated in Figure 2. The amplitude transmittance in the filter transparency as a function of the components of frequency $f_{x}$ and $f_{y}$ is proportional to $T_{\mathrm{A}}\left(\mathrm{f}_{\mathrm{x}}, \mathrm{f}_{\mathrm{y}}\right)$

$$
\begin{aligned}
=a & +2\left|H\left(f_{x}, f_{y}\right)\right| \cos \left[2 \pi b f_{x}+\arg H\left(f_{x}, f_{y}\right)\right] \\
=a & +2 \operatorname{Re}\left[H\left(f_{x}, f_{y}\right) \exp \left(2 \pi i b f_{x}\right)\right] \\
=a & +H\left(f_{x}, f_{y}\right) \exp \left(2 \pi i b f_{x}\right) \\
& +\bar{H}\left(f_{x}, f_{y}\right) \exp \left(-2 \pi i b f_{x}\right)
\end{aligned}
$$

where $a$ and $b$ are constants. The constant $b$ is the "frequency" (in the frequency domain) of the carrier, and the constant a is chosen so that $T_{A}$ is always positive.

It remains to discuss means of producing the hologram filter used above. A method of doing this for the case in which a point source is available for determining the optical transfer function will be discussed and is illustrated in Figure 3.

According to the discussion following equation (10), the optical transfer function G is 'simply the Fourier transform of the image of a point source, that is, the Fourier transform of the point spread function $\mathrm{g}$. The image of the point source can be transformed to $G$ by means of a lens, as previously described.

Now consider equation (5). The term $\bar{G}$ can be produced by making a hologram by causing a reference beam of collimated coherent light to interfere with the light forming $G$ in order to produce interference 
fringes which form the carrier wave. By suitably choosing the angle of the beam, the desired carrier frequency is selected, and by suitably choosing the polarity of the angle compared to the desired diffraction direction in Figure $2, \bar{G}$ is obtained instead of $G$.

For the moment neglect the term $\beta^{2}$ in the denominator of equation (5). To produce the function $1 /|G|^{2}$ it is necessary only to expose a negative in the ordinary way to the function $G$ and to develop it for a gamma of 2 , since the transmission through a developed negative is proportional to the minus gamma power of the exposure. If the above hologram containing $\vec{G}$ is simply stacked together with this negative containing $1 /|\mathrm{G}|^{2}$, the function $\mathrm{G} /|\mathrm{G}|^{2}=1 / \mathrm{G}$ is produced. This filter transfer function would restore the image completely in the absence of noise $(\beta=0)$.

To account for the presence of noise (and zeros in G) the term $B^{2}$ is inserted into the denominator of equation (5), as previously explained. The effect of this term can be approximated in a crude but sufficient way as follows. In making the negative for $1 /|\mathrm{G}|^{2}$ as described above, the exposure is adjusted so that for sufficiently small values of $G$ the negative becomes underexposed, and thus the amplitude transmission no longer follows $1 /|\mathrm{G}|^{2}$, but instead approaches a constant value corresponding to $1 / \beta^{2}$. The resulting negative is stacked with the hologram for $\bar{G}$ as before, to produce a filter that approximates the filter transfer function $\mathrm{H}$ as given by equation (5). This filter is used as previously shown in Figure 2 .

\section{DIGITAL TECHNIQUES}

Methods of using a digital computer to perform the image processing will now be perform the image processing will now method and the transform method will be described, as we 11 as the hybrid method, which is a combination of the other two.

\section{Convolution Method}

In the convolution method, equation (7), which represents a convolution in the space domain, is used. (The filter impulse response h is here usually known as the data multipliers.) For digital data, the convolution in equation (7) can be written out as follows:

$$
\begin{aligned}
& \hat{z}\left(k_{x}, k_{y}\right) \\
& =\sum_{k_{x}^{\prime}=-n_{x}}^{n_{x}} \sum_{k_{y}^{\prime}=-n_{y}}^{n_{y}} h\left(k_{x}^{\prime}, k_{y}^{\prime}\right) z\left(k_{x}-k_{x}^{\prime}, k_{y}-k_{y}^{\prime}\right)
\end{aligned}
$$

where the span of the filter impulse response has been constrained to a span of $2 n_{x}+1$ by $2 n_{y}+1$ points in order to keep the computation time down. ( $n_{x}$ and $n_{y}$ must be large enough to allow all of the important effects in the desired transfer function $\mathrm{H}$ to be approximated sufficiently we11.) In actual practice, the computations in equation (12) can sometimes be reduced by taking advantage of the symmetry, if any, of the data multipliers.

If the input image is read in and out of the computer by columns (y direction), it $c$ an be seen that it is necessary to store $2 \mathrm{n}_{\mathrm{x}}+1$ complete columns of the input image in the computer memory at any one time. In order to reduce the memory requirements, the following method was developed for use in the program OSIR on the IBM 7094 at the Eastern Test Range. These $2 n_{x}+1$ columns are stored in packed form, as 9-bit fixed-point values, four to a word. Then on $1 y$ the $2 n_{x}+1$ by $2 n_{y}+1$ points needed at a time in equation (12) are stored in normal unpacked form. Each time a new filtered point is computed, $2 \mathrm{n}_{\mathrm{x}}+1$ points are unpacked. This method is illustrated in Figure 4.

\section{Transform Method}

In the transform method, equation (6) is used, which represents a point-by-point multiplication in the frequency domain. The transformation of the image to the frequency domain and the transformation of the result back to the space domain after multiplication by the filter transfer function $H$ are performed by finite discrete two-dimensional Fourier transforms, which may be defined as follows:

$$
\begin{aligned}
& z\left(j_{x}, j_{y}\right) \\
& =\sum_{k_{x}=0}^{N_{x}-1} \sum_{k_{y}=0}^{N_{y}-1} z\left(k_{x}, k_{y}\right) \exp \left[2 \pi i\left(\frac{j_{x} k_{x}}{N_{x}}+\frac{j_{y} k_{y}}{N_{y}}\right)\right]
\end{aligned}
$$

$$
\begin{array}{r}
\hat{z}\left(k_{x}, k_{y}\right)=\frac{1}{N_{x}^{N}} \sum_{j_{x}=0}^{N_{x}-1} \sum_{j_{y}=0}^{N_{y}-1} \hat{z}\left(j_{x}, j_{y}\right) \\
x \exp \left[-2 \pi i\left(\frac{j_{x} k_{x}}{N_{x}}+\frac{j_{y} k_{y}}{N_{y}}\right)\right]
\end{array}
$$

where $\mathrm{N}_{\mathbf{x}}$ and $\mathrm{N}_{\mathrm{y}}$ represent the number of columns and rows, respectively, in the image.

The direct implementation of equations (13) and (14) would require too much computer time. Therefore, the Fast Fourier Transform developed by Cooley and 
Tukey (10) is used instead. Furthermore, large images may not fit into the computer memory. Therefore, a method of partitioning the image and using disk storage was developed for use in the program OSIP on the IBM $360 / 65$ at the Eastern Test Range. This method can be described as follows, provided that the image is available by columns (y direction).

The real array in the space domain is divided into groups of adjacent columns and groups of adjacent rows, as shown in Figure 5, such that one entire group of columns or two entire groups of rows can fit into the main memory at one time, together with the rest of the program. Each portion of the array that is common to a particular group of columns and a particular group of rows is called a block. Each group of columns is read into memory at one time and is transformed along the $y$ dimension only. Each block in this group is then read out separately to disk storage. After all of the groups of columns have been transformed in this way, the transforming of the rows begins. All of the blocks comprising one group of rows are read from disk storage into memory by using the direct-access feature of the disks, these rows are transformed along the $x$ dimension, and the result is read out to external storage (or used in subsequent processing) by rows. When all the groups of rows have been processed in this way, the two-dimensional transform of the original array is sitting in external storage, stored by rows (or has otherwise been used by rows). When an inverse transform is desired, the above operations are reversed, starting with the array stored by rows and ending with the array stored by columns. Thus the computations using the arrays can be arranged so that in the space domain any array is always stored by columns and in the frequency domain any array is always stored by rows. In the applications here, the arrays in the space domain will always be real, whereas in the frequency domain they will be complex. However, only the lower half of the array is used in the frequency domain, since, if $z(x, y)$ is real, then $z\left(N_{x}-j_{x}, N_{y}-j_{y}\right)$ $=Z\left(j_{x}, j_{y}\right)$.

The one-dimensional transforms along the columns or rows needed in the above method are performed by a subroutine written specifically for this task using the Fast Fourier Transform. This subroutine can handle arrays of sizes of the form $2^{n} \cdot 3^{m}$. (The image is filled out with a constant to the next larger size of this form in each dimension.) By using this subroutine and the above method of disk storage, a 1024 by 1024 array can be transformed in 10 minutes on the IBM $360 / 65$, when 41,000 words ( 164,000 bytes) of main memory for data and coding for this part of the program are used.

\section{Hybrid Method}

The hybrid method essentially consists of using the convolution method in one dimension and the transform method in the other dimension. That is, the image is transformed to the frequency domain along one dimension only (here assumed to be $y$ ), the result is convolved with the filter function along the other dimension $(x)$, and this result is transformed back to the space domain along the first dimension $(y)$. This can be stated mathematically as follows:

$\hat{g}\left(k_{x}, j_{y}\right)=\sum_{k_{y}=0}^{N_{y}-1} z\left(k_{x}, k_{y}\right) \exp \left(\frac{2 \pi i j_{y} k_{y}}{N_{y}}\right)$
$\hat{g}\left(k_{x}, j_{y}\right)=\sum_{k_{x}^{\prime}=-n_{x}}^{n_{x}} q\left(k_{x}^{\prime}, j_{y}\right) g\left(k_{x}-k_{x}^{\prime}, j_{y}\right)$
$\hat{z}\left(k_{x}, k_{y}\right)=\frac{1}{N_{y}} \sum_{j_{y}=0}^{N_{y}-1} \hat{g}_{\left(k_{x}, j_{y}\right) \exp \left(-\frac{2 \pi i j_{y} k_{y}}{N_{y}}\right)}$

The filter function \& needed above could be obtained from the equivalent data multipliers that would be used in the convolution method, as follows:

$H\left(k_{x}, j_{y}\right)=\sum_{k_{y}=-n_{y}}^{n_{y}} h\left(k_{x}, k_{y}\right) \exp \left(\frac{2 \pi i j_{y} k_{y}}{N_{y}}\right)$

The script letters used in the above four equations represent quantities expressed in a mixture of the space domain ( $\mathrm{k}$ index) and the frequency domain ( $j$ index).

The one-dimensional Fourier transforms denoted by equations $(15),(17)$, and (18) would actually be performed by the Fast Fourier Transform. Furthermore, since $z\left(k_{x}, N_{y}-j_{y}\right)=z\left(k_{x}, j_{y}\right)$ (and similarly for $f$ and $t^{\prime}$ ), only half-sized arrays are used in the mixed domain, in order to save more time and storage.

Although the hybrid method has never actualiy been used to the author's know1edge, calculations indicate that in some cases it would require less computer time than either of the other two methods. Furthermore, the hybrid method is suitable in some cases in which the transform method is completely impractical due to the lack of disk storage and the size of the computer memory. However, for large filter spans the hybrid method requires a larger main memory than does the convolution method or the transform method with disk storage. 
In Figure 6 the approximate amount of IBM $360 / 65$ computer time required per image point to process a single image by each of the three methods is shown as a function of the span of the filter impulse response (assumed to be square). These times are rough estimates assuming efficient programming and typical conditions suitable for the method at hand (for example, the use of disk storage for the transform method). The two dashed lines show special cases in which the convolution method can be made faster. For the curve marked $S$ it is assumed that the desired filter transfer function $\mathrm{H}$ is real and is a function only of the magnitude of the frequency and thus the impulse response also has this symmetry about the origin. For the curve marked D it is assumed that $\mathrm{H}$ can be factored into separate functions of $f_{x}$ and $f_{y}$ and that these functions are symmetrical about the origin. (While the $\mathrm{S}$ case is fair $1 \mathrm{y}$ common, the D case is not.)

\section{Computation of Filter}

The digital techniques of performing the actual filtering of an image have just been described. However, the computing of the filter itself (performed before the actual filtering) involve some special considerations depending on the digital method being used and on the method of determining the optical transfer function. Here it will be assumed that the optical transfer function is being obtained from a known object according to equation $(10)$, and the particular techniques for implementing the computations for each of the three methods will be discussed.

In any of the methods, if a known object has been photographed as previously described and the results digitized, equation (10) can be used to compute the optical transfer function. To do this, the known image $\hat{u}$ and its biurred form $u$ in digital form representing intensity would each be processed as follows. The background intensity level (if any) is subtracted from each point in the image, so that the results indicate 1 ight from the known object only. The image is truncated to an area sufficiently large to include the significant information, but no larger, in order to reject as much noise as possible.

In the convolution method, the computations now proceed as follows. The truncated images $u$ and $\hat{u}$ are filled out with zeros to produce arrays of the size decided on for $h$. The finite discrete two-dimensional Fourier transforms of $u$ and $\hat{u}$ are then computed to obtain $U$ and $U$. The ratio of these at each point is computed to obtain the optical transfer function $G$, as stated by equation (10). Once the optical transfer function is available, the desired transfer function $\mathrm{H}^{\prime}$ of the filter to be used is computed at each point in the frequency domain using equation (5). (The prime is used on $H$ here because this transfer function may not be achieved exactly, as is explained below.) Then the inverse finite discrete two-dimensional Fourier transform of $\mathrm{H}^{\prime}$ is computed to obtain $h^{\prime}$. This function could be used as the impulse response $h$ of the filter. However, since it has been necessary to limit h to a fairly small array for computation purposes, small values of the ideal h may exist outside of this array. By limiting the size of the array, these values rejected have in effect been replaced by zeros (and the values within the array have adjusted themselves also). Thus a discontinuity has been introduced into the function h, which would cause "ringing" in the frequency domain. (That is, if h' were filled out with zeros to form an array the size of the image $z$ and the result transformed to the frequency domain, the resulting function $\mathrm{H}^{\prime}$ would be identical to the function $\mathrm{H}^{\prime}$ above at those frequencies which were defined above as points in the array, but with the fullsized array, many points of frequency have been added between these original cnes, and the value of $\mathrm{H}^{*}$ at these points would oscillate about a smooth curve passing through the original values.) Thus it may be desirable to multiply $h^{\prime}$ by some apodizing function, which decays gradually to zero at the edges, to obtain $h$, in order to remove the discontinuity.

The above computation of $h$ does not require much computer time, especially if the Fast Fourier Transform is used, since the arrays to be transformed are small. (They are the same size as $h$.) The function $h$ forms the filter data multipliers to be used in equation (12), as previously described.

In the transform method, one way of computing the filter transfer function $\mathrm{H}$ is first to compute the filter impulse response h exactly as it would be computed in the convolution method, as described above. Then h is filled out with zeros to make an array the same size as the image, and this full-sized array is transformed to the frequency domain by the same two-dimensional Fourier transform subroutine used to transform $z$. According to the convolution theorem, the transform method in this case would give results that are mathematica11y identical to those that would be obtained with the convolution method. However, it will be recalled that in the convolution method it was necessary to truncate $h$ to a reasonably small span in order to save computation time in the convolution. Since this is no longer necessary in the transform method, it may be desirable to 
remove this truncation. Doing so will often produce slightly more accurate results, since, even though the original point spread function $g$ may be limited to a small span, the impulse response $h$ needed to perform the inverse of $\mathrm{g}$ will usually not be so limited. Therefore, the following method of computing $\mathrm{H}$ may be preferable.

In this alternate technique for use with the transform method, the computations proceed as follows, starting with the truncated images $u$ and $u$ previously described for the convolution method. The images $u$ and $\hat{u}$ are filled out with zeros to produce arrays of the size of the image $z$. The same two-dimensional Fourier transform subroutine used to transform the image $z$ to the frequency domain is then used to transform $u$ and $\hat{u}$ to the frequency domain to obtain the arrays $U$ and $U$. (As previously explained, these arrays in the frequency domain need to be only half of the full size.) The ratio of these at each point is computed to obtain the optical transfer function $G$, as stated by equation (10). (In the next subsection a way of saving time in these computations is described.) Once the optical transfer function is available, the transfer function $H$ of the filter to be used is computed at each point in the frequency domain using equation (5). The resulting transfer function is used in equation (6), as previously described.

For the hybrid method, the filter can be obtained from the corresponding filter for the convolution method by using equation (18), as previously described.

Transforming Two Arrays at Once

In the second method of computing the filter for use with the digital transform method as discussed above, it was seen that the arrays $u$ and $\hat{u}$ had to be transformed to the frequency domain to obtain U and $\hat{0}$. Thus it might seem that two ful1sized two-dimensional transforms must be computed. However, computation time can be saved by utilizing the fact that, if the images $u$ and $\hat{u}$ are much smaller than the image $z$, as is usually the case, it is possible to transform them both at once. This would be done by the following method.

The images $u$ and $\hat{u}$ are combined into a single array the size of $z$ (after $z$ has been filled out, if necessary, to a size that can be transformed) by centering one of them ( $\hat{u})$ on the $y$ axis ( $k_{x}=0$ line), wrapped around the edge of the array, and centering the other one (u) half-way around the array (considered cyclicly) from this (centered on the $\mathrm{k}_{\mathrm{x}}=\mathrm{N}_{\mathrm{x}} / 2$ line), as shown in Figure 7 . The rest of the array is filled in with zeros. This array, called $w$, is then transformed to the frequency domain to obtain the array $W$. W can be filtered in order to separate the two arrays $\mathrm{U}$ and $\hat{\mathrm{U}}$. Since $\mathrm{u}$ and $\hat{\mathrm{u}} \mathrm{can}$ be separated from $w$ by multiplying by a function of $x$, by the convolution theorem a one-dimensional convolution (in the $x$ direction) of W with suitable functions can separate $U$ and 0 . The span of the function chosen to convolve with $W$ must be short, however, in order to keep the computation time low, especially since $W$ is complex. This restriction prevents a complete separation of $\mathrm{U}$ and $\mathrm{U}$; however, the separation can be made sufficiently complete for most practical purposes. To avoid distortion due to the limitations of the filter, $u$ and $\hat{u}$ each can be divided by the Fourier transform of the appropriate function chosen to convolve with W, before they are used in the above, in order to compensate for the response of the filter. (This technique of avoiding distortion can be called pre-emphasis.)

One possible choice of filtering to use for the above separation is the following (where the index $j_{x}$ is considered to "wrap around" the array cyclicly, modulo $\mathrm{N}_{\mathbf{x}}$ ):

$$
\begin{aligned}
& \hat{U}\left(j_{x}, j_{y}\right)=(-1)^{j_{x}} \sum_{j^{\prime}=-3}^{3} R\left(j^{\prime}\right) W\left(j_{x}-j^{\prime}, j_{y}\right) \\
& U\left(j_{x}, j_{y}\right)=\sum_{j^{\prime}=-3}^{3}(-1)^{j^{\prime}} R\left(j^{\prime}\right) W\left(j_{x}-j^{\prime}, j_{y}\right)
\end{aligned}
$$

where

$$
\begin{aligned}
& \mathrm{R}(0)=.302812500 \\
& \mathrm{R}( \pm 1)=.231953125 \\
& \mathrm{R}( \pm 2)=.098593750 \\
& \mathrm{R}( \pm 3)=.018046875
\end{aligned}
$$

Pre-emphasis can be achieved by dividing both $\hat{\mathrm{u}}\left(\mathrm{k}^{\prime}, \mathrm{k}_{\mathrm{y}}\right)$ and $\mathrm{u}\left(\mathrm{k}^{\prime}, \mathrm{k}_{\mathrm{y}}\right)$ by $\mathrm{r}\left(\mathrm{k}^{\prime}\right)$ before they are combined into $w$, where $k^{\prime}$ is here assumed to be zero at the center of each of these images $\left(k_{x}=0\right.$ and $\mathrm{k}_{\mathrm{x}}=\mathrm{N}_{\mathrm{x}} / 2$ in $\mathrm{w}$, as explained above), and where

$r\left(k^{\prime}\right)=\sum_{j^{\prime}=-3}^{3} R\left(j^{\prime}\right) \cos \frac{2 \pi j^{\prime} k^{\prime}}{N_{x}}$

If the above seven-point filter is used with pre-emphasis, $U$ and 0 will be separated with no distortion and less than .0006 amplitude cross-talk if $u$ and $\hat{\mathrm{u}}$ each have a width less than one fourth of the width of the full-sized array. 


\section{EXAMPLE}

An example of image enhancement using the digital transform method will now be presented.

Figure 8 shows the test target that was used. This was photographed deliberately out of focus to produce the blurred image shown in Figure 9. The negative from which Figure 9 was made was scanned in a microdensitometer. A sample interval of 1 millimeter was used in each dimension, with a.1 millimeter square slit. By using the characteristic curve of the emulsion, the measured density values were converted to intensity in arbitrary units such that the white areas in the scene have the approximate value 1 . The resulting values were plotted using a variable intensity plot on a cathode-ray tube plotter to produce Figure 10. No filtering was done in this case. (Figure 10 is shown for comparison with later figures; it should look essentially the same as Figure 9.)

Threshold plots, in which every intensity above or below a certain threshold is plotted as all white or all black, respectively, were also made, for maximum contrast enhancement. The same blurred image shown in Figure 10 is plotted at two different thresholds in Figures 11 and 12 . In none of these are the numerals present in the original scene readable.

In order to determine the optical transfer function, the vertical and horizontal bars in Figure 8 were used as known objects. The regions of the data in Figure 10 containing these bars were used in accordance with equation (10) to obtain a raw estimate. Furthermore, since the blurring was caused by an out of focus camera, the shape of the transfer function was computed theoretically by assuming a circular aperture. The frequency scale factor of the theoretical function was obtained by adjusting it to fit the raw estimate. The resulting estimate of the optical transfer function $G$ is shown in Figure 13, which is assumed to apply in a11 directions in the twodimensional frequency domain. This was used in euation (5) with $\beta=.025$ to produce the filter transfer function $H$ shown in Figure 14.

The image shown in Figure 10 was filtered by this filter using the transform method, and the result is shown in Figure 15 .

Threshold plots of this same filtered image are shown in Figures 16 and 17 . The large and medium-sized numerals can now be read. The small numerals show up faintly, but in order for them to be readable a smaller sample interval than 1 millimeter would probably be required. The circular patterns visible in the filtered image are due to specks of dirt (or other imperfec- tions) on the original out of focus negative. It is possible to correct partially these erroneous points by an editing procedure before the image is filtered. The result of filtering such edited data is shown in Figure 18 .

\section{NOMENCLATURE}

a,b - arbitrary constants.

$f_{x} \quad-x$ component of frequency.

$f_{y} \mathbf{x} \quad-y$ component of frequency.

g - optical impulse response (point spread function).

G - optical transfer function ( $g$ transformed to frequency domain).

h - filter impulse response (data multipliers for digital filter).

$\mathrm{H}$ - filter transfer function (h transformed to frequency domain).

4 - filter expressed in mixed domain.

i $-\sqrt{-1}$.

$j \quad-x$ frequency component integer index $\left(=N_{x} \Delta x f_{x}\right)$, used modulo $N_{x}$.

$j_{y} \quad-y$ frequency component integer index $\left(=N_{y} \Delta f_{y}\right)$, used modulo $N_{y}$.

$\mathrm{k}_{\mathrm{x}} \quad-\mathrm{x}$ dimension integer index $(=\mathrm{x} / \Delta \mathrm{x})$, used modulo $\mathrm{N}_{\mathbf{x}}$.

$\mathrm{k}_{\mathrm{y}} \quad-\mathrm{y}$ dimension integer index $(=y / \Delta y)$, used modulo $\mathrm{N}_{\mathrm{y}}$.

L - focal length of lens.

$\mathrm{m}, \mathrm{n}$ - arbitrary integers.

$n_{x}, n_{y}$ - filter semi-span in $x$ or $y$ dimension in digital convolution method.

$\mathrm{N}_{\mathrm{x}}, \mathrm{N}_{\mathrm{y}}$ - number of columns or rows in digitized image (after being filled out to a size that can be transformed).

$\mathrm{P}$

power spectrum of signal denoted by subscript.

$r, R$ - filter used to separate $U$ and $\hat{U}$ out of $W$.

$\mathrm{T}_{\mathrm{A}} \quad$ - amplitude transmittance of analog filter transparency.

u

- intensity in image of known object.

- image $u$ transformed to frequency domain.

- combined images $u$ and $\hat{u}$.

W

- w transformed to frequency domain.

$\mathrm{x}$

- horizontal dimension in image.

$y$

z

Z
- vertical dimension in image.

- intensity in image to be processed.

- image $z$ transformed to frequency domain. 


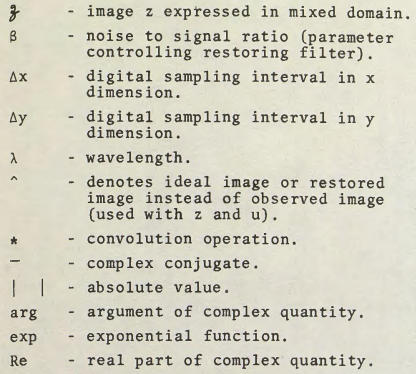

\section{ACKNOWLEDGMENTS}

The analog techniques described herein were developed primarily by various others $(1,2,3,1,1,12)$. In developing the digital techniques and in preparing the example the author received help and programming services from many of his coworkers at RCA. Among these were Anne Exline, Dennis Johnston, Sheldon Jordan, Dave Karlson, Robert Lastra, John Rollins, Neil Walker, and Larry Wilson.

\section{REFERENCES}

(1) P. E1ias, D. S. Grey, and D. Z . Robinson, "Fourier Treatment of Optica1 Processes," Journal of the optical society of America, Vo1. 42, No. 2, pp. 127-134, February 1952.

(2) E. L. O'Nei11, "Spatial Filtering in Optics," IRE Transactions on Information Theory, Vol. IT-2, No. 2, pp. 56-65, June 1956 .

(3) L. J. Cutrona, E. N. Leith, C. J. Palermo, and L. J. Porce11o, "Optica1 Data Processing and Filtering Systems," IRE Transactions on Information Theory, Vo1. IT-6, No. 3, pp. 386-400, June 1960.

(4) A. Vander Lugt, F. B. Rotz, and A. Klooster, Jr., "Character-Reading by Optical Spatial Filtering," Optical and Electro-optical Information Processing, ed. J. T. Tippett et al. The Massachusetts Institute of Technology Press, 1965.

(5) J. L. Harris, Sr., "Image Evaluation and Restoration," Journal of the optical Society of America, Vol. 56 , No. 5, pp. 569-574, May 1966 .
(6) B. L. McGlamery, "Restoration of Turbulence-Degraded Images," Journal of the optical Society of America, Vol. 57 , No. 3, pp. 293-297, March 1967.

(7) L. A. Wainstein and V. D. Zubakov, Extraction of Signals from Noise, trans. R. A. Silverman, Prentice-Ha11, 1962.

(8) K. Preston, Jr., "Use of the Fourier Transformable properties of Lenses for Signal Spectrum Analysis," optical and Electro-optical Information Processing, ed. J. T. Tippett et al., The Massachusetts Institute of Technology Press, 1965.

(9) E. N. Leith and J. Upatnieks, "Wavefront Reconstruction with Continuous-Tone objects," Journal of the optioal Society of America, Vo1. 53, No. 12, pp. 1377-1381, December 1963.

(10) J. W. Cooley and J. W. Tukey, "An Algorithm for the Machine Calculation of Complex Fourier Series," Mathematics of Computation, Vo1. 19, No. 90, pp. 297-301, Apri1 1965.

(11) E. N. Leith, A. Kozma, and J. Upatnieks, "Coherent Optical Systems for Data Processing, Spatial Filtering, and Wavefront Reconstruction," optioat and Electro-Optical Information Processing, ed. J. T. Tippett et $a l$., The Massachusetts Institute of Technology Press, 1965.

(12) G. W. Stroke, G. Indebetouw, and C. Puech, "A Posteriori Holographic Sharp-Focus Image Restoration from Ordinary Blurred Photographs of ThreeDimensional objects Photographed in Ordinary White Light," Phyoica Letters, Vol. 26A, No. 9, pp. 443-444, 25 March 1968. 


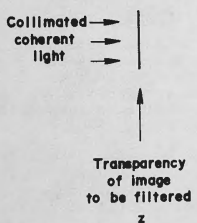

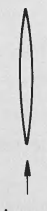

Lens I
Transparency of filter transfer function H

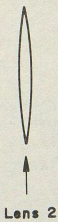

Filtered Image

Figure 1

optigal filteping of image

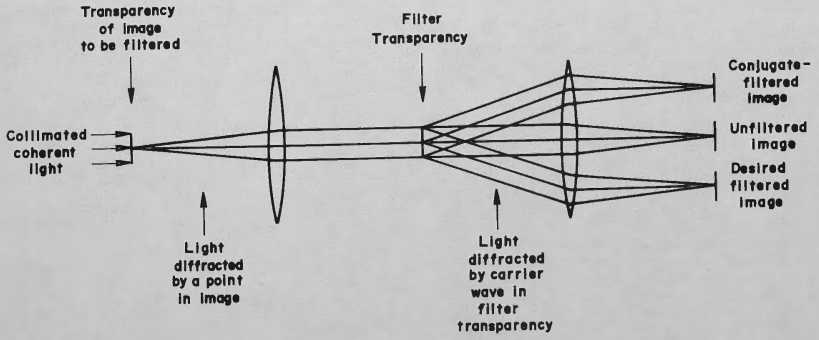

Figure 2 


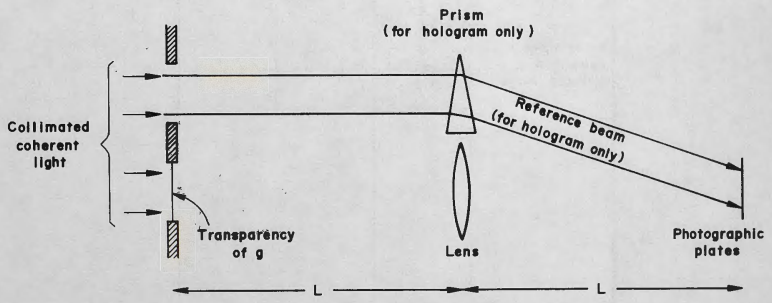

Figure 3

PRODUCTION OF TWO PLATES FOR COMPLEX FILTER 


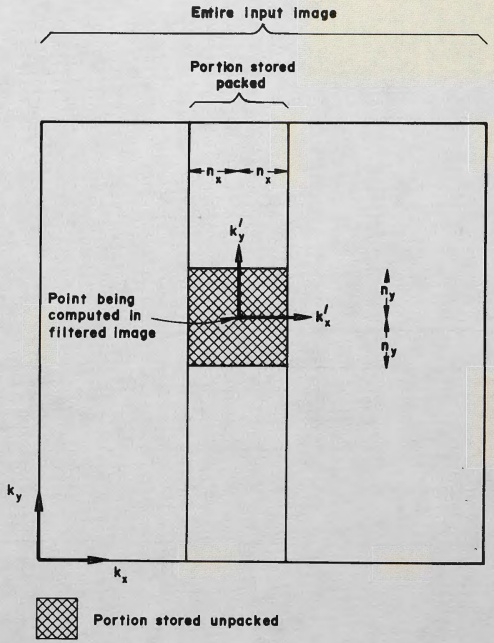

Figure 4

BTORAGE OF DATA FOR CONVOLUTION METHOD 


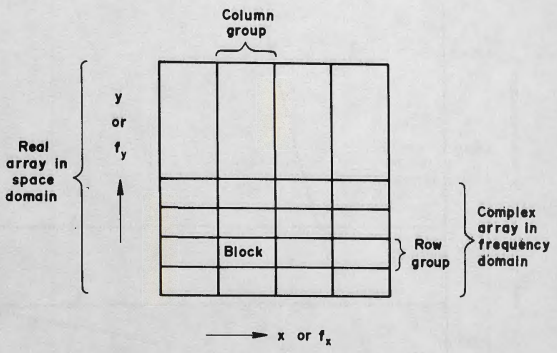

Figure 5

PARTITIONING OF ARRAY FOR TRANBFORMATION 


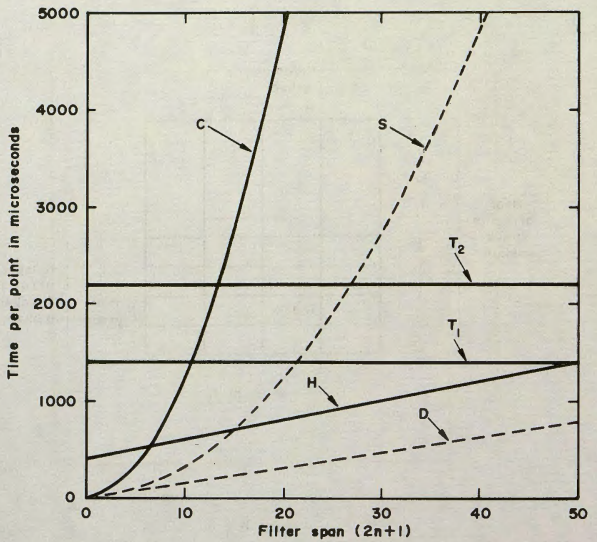

$T_{1}$ - transform method (excluding filter computation)

C - convolution method

$T_{2}$-transform method (including filter computation)

$S$ - convolution method with circular symmetry

H - hybrid mothod

D - convolution using two one-dimensional symmetrical direct fllters

Figure 6

ESTIMATED Computation TINE ON IEM seq/s 


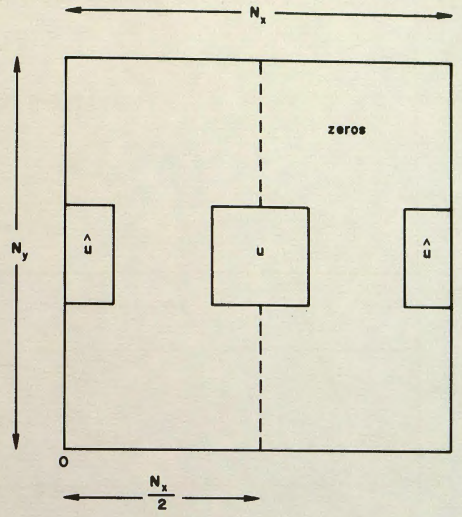

Figure 7

Foncation of w AnRay 


\section{5 \\ 409 \\ 586}

Figure 8. Original Scene.

\subsection{0}

Figure II. Unfiltered Image, Threshold .5.

Figure 9. Blurred Photograph.
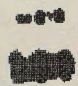

Figure 12. Unfiltered Image, Threshold .7.

Figure 10. Unfiltered Image, Variable Intensity Plot 


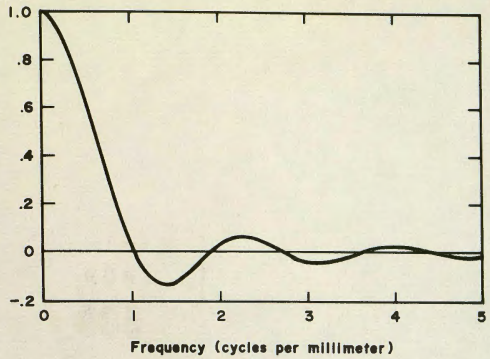

Figure 13

OPTICAL TRANGFER FUNCTIOM IN EXAMPLE

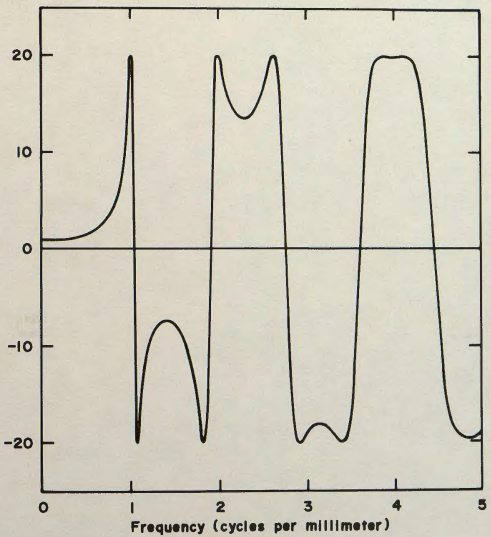

Figure 14

FILTER TRAMGFER FUNCTION IM EXAMPLE 


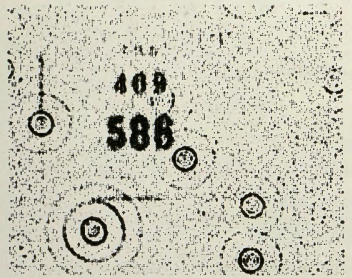

Figure I5. Filtered Image, V:sifable Intensity Plot.

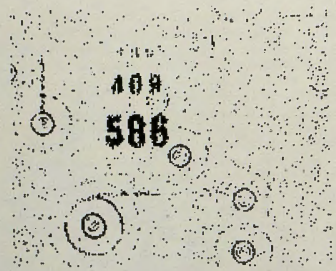

Figure 16. Filtered Image, Threshold .5.

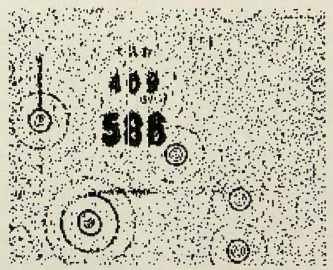

Figure 17. Filtered Image, Threshold ,7. 